\title{
Nine Years of Cosmic Rays Investigation by the PAMELA experiment
}

\footnotetext{
M. Boezio*1 , M. Martucci ${ }^{4,2}$, A. Bruno ${ }^{6}$, V. Di Felice ${ }^{8,5}$, R. Munini ${ }^{1,9}$, O. Adriani ${ }^{11,12}$, G. C. Barbarino ${ }^{13,14}$, G. A. Bazilevskaya ${ }^{3}$, R. Bellotti ${ }^{6,16}$, E. A. Bogomolov ${ }^{17}$, M. Bongi $^{11,12}$, V. Bonvicini ${ }^{1}$, S. Bottai ${ }^{12}$, F. Cafagna ${ }^{16}$, D. Campana ${ }^{14}$, P. Carlson ${ }^{19}$, M. Casolino $^{8,20}$, G. Castellini ${ }^{21}$, C. De Donato ${ }^{4,8}$, C. De Santis ${ }^{8}$, N. De Simone ${ }^{8}$, V. Formato $^{1,9, x}$, A. M. Galper ${ }^{18}$, A. V. Karelin ${ }^{18}$, S. V. Koldashov ${ }^{18}$, S. Koldobskiy ${ }^{18}$, S. Y. Krutkov $^{17}$, A. N. Kvashnin ${ }^{5}$, A. Leonov ${ }^{18}$, V. Malakhov ${ }^{18}$, L. Marcelli ${ }^{4}$, A. G. Mayorov $^{18}$, W. Menn ${ }^{22}$, M. Mergè ${ }^{4,8}$, V. V. Mikhailov ${ }^{18}$, E. Mocchiutti ${ }^{1}$, A. Monaco ${ }^{6,16}$, N. Mori ${ }^{12,7}$, G. Osteria ${ }^{14}$, F. Palma ${ }^{4,8}$, B. Panico ${ }^{14}$, P. Papini ${ }^{12}$, M. Pearce ${ }^{19}$, P. Picozza $^{4,8}$, M. Ricci' ${ }^{2}$, S. B. Ricciarini ${ }^{12,21}$ R. Sarkar ${ }^{10}$, V. Scotti ${ }^{13,14}$, M. Simon ${ }^{22}$, R. Sparvoli ${ }^{4,8}$, P. Spillantini ${ }^{11,12}$, Y. I. Stozhkov ${ }^{5}$, A. Vacchi ${ }^{1}$, E. Vannuccini ${ }^{12}$, G. Vasilyev $^{17}$, S. A. Voronov ${ }^{18}$, Y. T. Yurkin ${ }^{18}$, G. Zampa ${ }^{1}$ and N. Zampa ${ }^{1}$

${ }^{1}$ INFN, Sezione di Trieste, I-34149 Trieste, Italy

2 INFN, Laboratori Nazionali di Frascati, Via Enrico Fermi 40, I-00044 Frascati, Italy

${ }^{3}$ Lebedev Physical Institute, RU-119991 Moscow, Russia

4 Department of Physics, University of Rome "Tor Vergata" I-00133 Rome, Italy
5 Agenzia Spaziale Italiana (ASI) Science Data Center, I-00044 Frascati, Italy

${ }^{6}$ University of Bari, I-70126 Bari, Italy

${ }^{7}$ Centro Siciliano di Fisica Nucleare e Struttura della Materia (CSFNSM), Viale A. Doria 6, I-95125 Catania, Italy

${ }^{8}$ INFN, Sezione di Rome "Tor Vergata", I-00133 Rome, Italy

${ }^{9}$ University of Trieste, Department of Physics, I-34147 Trieste, Italy

10 Indian Centre for Space Physics, 43 Chalantika, Garia Station Road, Kolkata 700084, West Bengal, India

${ }^{11}$ University of Florence, Department of Physics, I-50019 Sesto Fiorentino, Florence, Italy

12 INFN, Sezione di Florence, I-50019 Sesto Fiorentino, Florence, Italy

${ }^{13}$ University of Naples "Federico II", Department of Physics, I-80126 Naples, Italy

${ }^{14}$ INFN, Sezione di Naples, I-80126 Naples, Italy

${ }^{15}$ Lebedev Physical Institute, RU-119991, Moscow, Russia

${ }^{16}$ INFN, Sezione di Bari, I-70126 Bari, Italy

${ }_{17}^{1}$ Ioffe Physical Technical Institute, RU-194021 St. Petersburg, Russia

18 National Research Nuclear University MEPhI, RU-115409, Moscow, Russia

${ }^{19}$ KTH, Department of Physics, and the Oskar Klein Centre for Cosmoparticle Physics, AlbaNova University Centre, SE-10691 Stockholm,

Sweden

20 RIKEN, Advanced Science Institute, Wako-shi, Saitama, Japan

${ }^{21}$ IFA C, I-50019 Sesto Fiorentino, Florence, Italy

${ }^{22}$ Universitat Siegen, Department of Physics, D-57068 Siegen, Germany

${ }^{x}$ now at INFN, Sezione di Perugia, I-06123 Perugia, Italy

E-mail: mirko.boezio@ts.infn.it
} 


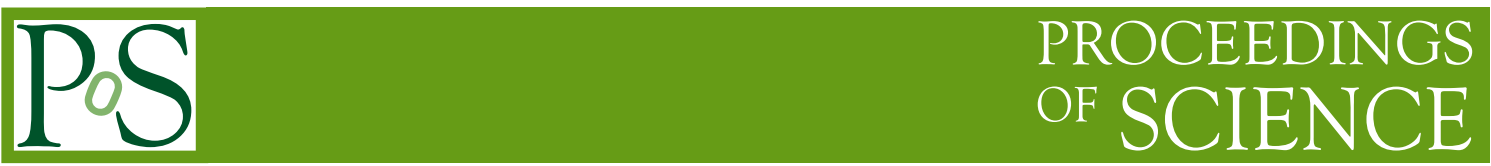

The PAMELA cosmic ray detector was launched on June $15^{\text {th }}$ of 2006 on board the Russian Resurs-DK1 satellite and during nine years of continuous data-taking it has observed very interesting features in cosmic rays, namely in the fluxes of protons, helium and electrons. Its discoveries might change our basic vision of the mechanisms of production, acceleration and propagation of cosmic rays in the Galaxy. Moreover, PAMELA measurements of cosmic antiproton and positron fluxes and positron-to-all-electron ratio have been setting strong constraints to the nature of Dark Matter. Measurements of boron, carbon, lithium and beryllium (together with the isotopic fraction) will also shed new light on the elemental composition of the cosmic radiation. Search for signatures of more exotic processes (such as the ones involving Strange Quark Matter) is also pursued. Furthermore, the instrument is still functional allowing a constant monitoring of the solar activity during its maximum and a detailed and prolonged study of the solar modulation, improving the comprehension of the heliosphere mechanisms. PAMELA is also measuring the radiation environment around the Earth, and it detected for the first time the presence of an antiproton radiation belt surrounding our planet. In this highlight paper PAMELA main results will be reviewed.

The 34th International Cosmic Ray Conference, 30 July- 6 August, 2015

The Hague, The Netherlands

\footnotetext{
${ }^{*}$ Speaker.
} 


\section{The PAMELA mission}

The PAMELA (a Payload for Antimatter-Matter Exploration and Light-nuclei Astrophysics) satellite experiment is designed to study the charged component of the cosmic radiation, focusing on antiparticles. PAMELA was launched with a Soyuz-U rocket on June $15^{\text {th }}$ of 2006 from the Baikonur cosmodrome (Kazakhstan). The apparatus is hosted on the Russian Resurs-DK1 satellite, a commercial Earth-observation spacecraft. At first the orbit was elliptical (altitude varying between 355 and $584 \mathrm{~km}$ ) and semipolar (inclination of about $70^{\circ}$ ) and with a period of about 94 minutes, see Fig 1 (left). In 2010 the orbit was set to be circular with an almost fixed altitude of about $550 \mathrm{~km}$. The mission was planned to last 3 years, but after 9 years of data taking it is still operational. This experiment could be considered as the greatest effort of the WiZARD Collaboration which, starting almost 30 years ago with the New Mexico State University group led by the late Robert L. Golden (July $28^{\text {th }} 1940$ - April $7^{\text {th }} 1995$ ), has successfully built and flown several balloon-borne experiments like MASS-89, MASS-91, Tramp-Si93, CAPRICE-94 and CAPRICE-98 and also satellites like NINA-1 (1998) and NINA-2 (2000). The WiZARD group has also pioneered some innovative detection techniques such as electromagnetic calorimeters, superconducting magnet spectrometers and ring imaging Čerenkov detectors in balloon flights [1]. The PAMELA main scientific objectives are to measure the antiproton spectrum up to $200 \mathrm{GeV}$, the positron spectrum up to $200 \mathrm{GeV}$, the electron spectrum up to $600 \mathrm{GeV}$, the proton and helium nuclei spectra up to 1.2 and $0.6 \mathrm{TeV} / \mathrm{n}$ respectively and the nuclei spectra (from Li to O) up to $\sim 100 \mathrm{GeV} / \mathrm{n}$ but also to search for antinuclei (with a $\overline{\mathrm{He}} / \mathrm{He}$ sensitivity of $10^{7}$ ), new forms of matter, e.g. strangelets and finally to detect possible structures in cosmic ray spectra from e.g. Dark Matter or new astrophysical sources. Furthermore PAMELA is well suited to conduct studies of cosmic-ray acceleration and propagation mechanisms in the Galaxy, solar modulation effects, the emissions of Solar Energetic Particles (SEPs) inside the heliosphere and investigate the particles in the Earth's magnetosphere.

\section{The instrument}

Space-borne missions have many benefits with respect to balloon-borne ones, like longer data taking time, no residual atmosphere affecting measurements or possibility to probe spectra at different magnetic rigidity cutoffs, but there are several stringent requirements that have to be taken into account. There are in fact some limitations on the maximum size, weight and on the power available for the on-board instruments; all the sub-detectors have to be designed in order to withstand the mechanical stresses during the launch as well as the radiation damage due to the extraterrestrial environment [1]. For these reasons the PAMELA detector was equipped with various instruments which guaranteed a great redundancy in case of unexpected malfunctioning of some sub-detectors amd allowed to perform in-flight calibrations, evaluate efficiencies and provide different measurements of a single event. The detector is composed of the following systems: 

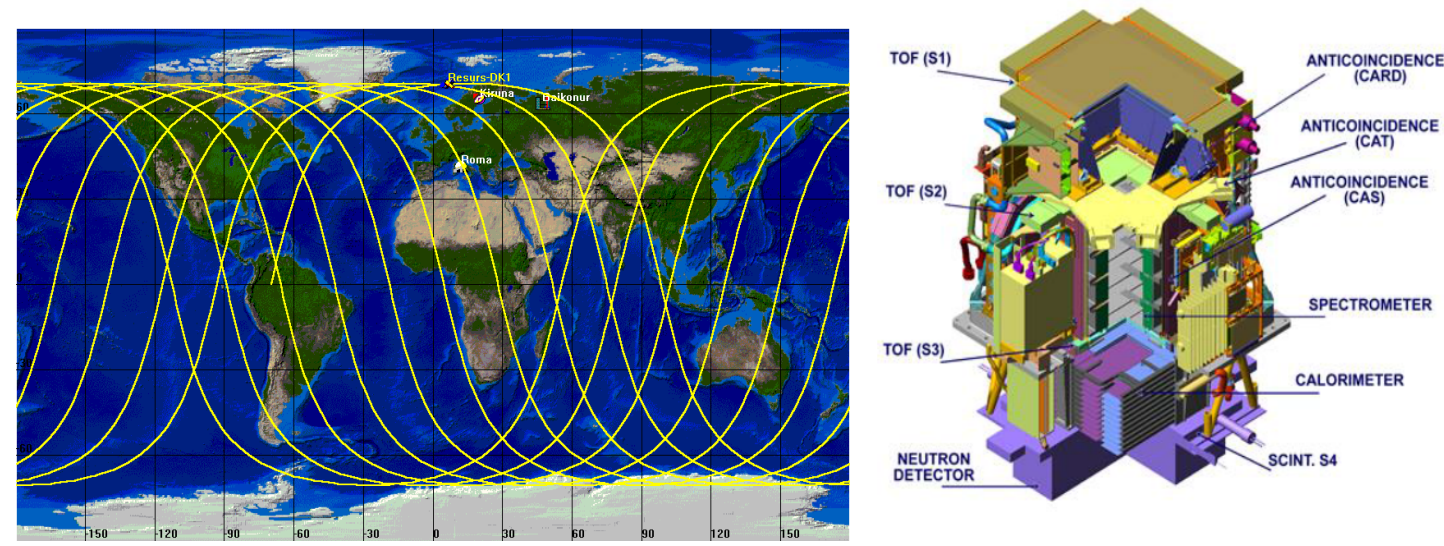

Figure 1: Orbit of PAMELA (left) and a scheme of the detector (right) with all the sub-detectors.

- a Time-of-Flight (ToF) system, with segmented scintillator planes divided into 3 groups $\left(S_{1}, S_{2}, S_{3}\right)$.

- a magnetic spectrometer to measure trajectories of the incoming particles

- an anticoincidence system with solid scintillators (CARD, CAT, CAS)

- an electromagnetic sampling calorimeter

- a shower tail-catcher scintillator

- a neutron detector

In Fig 1 (right) a sketch of the isometric view of the whole detector with all the subdetetctors is shown.

The core of the instrument is a Nd-Fe-B alloy permanent magnet (with residual magnetization of $1.3 \mathrm{~T}$ ) with a microstrip silicon tracking system composed of 6 equidistant $300 \mu \mathrm{m}$ thick planes that constitute a magnetic spectrometer used to determine the rigidity $(R=p c / Z e)$ and the charge $Z$ of particles crossing the cavity. The double-sided silicon sensors provide two independent impact coordinates on each plane and the blocks of the magnet are configured to provide an almost uniform magnetic field oriented along the $y$ direction inside a cavity $\left(13.1 \times 16.1 \mathrm{~cm}^{2}\right)$. The dimensions of the permanent magnet define the maximum geometrical factor of the PAMELA experiment to be $21.6 \mathrm{~cm}^{2} \mathrm{sr}$. A set of scintillators forms the anticoincidence system, which consists of 4 plastic scintillators (CAS) surrounding the sides of the magnet, one covering the top (CAT) and a set of 4 plastic scintillators (CARD) that surrounds the volume between the first two Timeof-Flight planes. An electromagnetic imaging calorimeter, placed under the spectrometer, comprises 44 single-sided silicon sensor layers (380 $\mu$ m thick) interleaved with 22 plates of tungsten absorber. It can distinguish between leptons and hadrons through the analysis of the shape of the shower they produce. It also measures the energy of electrons 
and positrons interacting in its volume and check the alignment of the tracker sensors. A Time-of-Flight system, composed of 6 layers of fast plastic scintillators segmented into orthogonal bars and arranged in 3 double planes $\left(S_{1}, S_{2}\right.$ and $\left.S_{3}\right)$ provides information on particle velocity $\beta$ and trigger. Both ends of each scintillator bar are glued to a plastic light guide which is mechanically coupled to a photomultiplier. An additional plastic scintillator $\left(S_{4}\right)$ is used to detect charged particles in case a shower is not completely contained in the calorimeter; it consists of a single square piece of $1 \mathrm{~cm}$ thick scintillator which is read out by 6 PMTs. Finally a neutron detector (36 proportional counters, filled with ${ }^{3} \mathrm{He}$ surrounded by a polyethylene moderator enveloped in a cadmium layer) at the bottom of the instrument complements the calorimeter in lepton-hadron discrimination. The entire apparatus is $\sim 1.2 \mathrm{~m}$ high, has a mass of $470 \mathrm{~kg}$ and an average power consumption of 355 W. More details on the instrument can be found in Ref [1].

\section{Protons, helium nuclei and electron spectra}

Hydrogen and helium are the most abundant components of galactic cosmic rays, constituting about $98 \%$ of the total flux and they are believed to be of primary origin. The energy spectra of hydrogen and helium have been measured by many experiments over the years. The first high-statistics and high-precision measurements, carried on by PAMELA from July 2006 to March 2008, are shown in Fig 2 (left) together with results of other experiments $[2,1]$. Interesting spectral features were found in the data, with significant implications for the understanding of high-energy galactic processes. The PAMELA data presented in Fig 2 (left) reveal a hardening in the spectrum of both $\mathrm{H}$ and He. These results are in good agreement with previous results below $100 \mathrm{GeV} / \mathrm{n}$ and consistent with the spectral features of the measurements by ATIC-2 and CREAM [1] at higher energies. Below $10 \mathrm{GeV} / \mathrm{n}$ discrepancies are evident, but this can be ascribed to different epochs of solar activity. Both spectra show deviations from a single power law model, exhibiting a spectral hardening at a rigidity of about $230-240 \mathrm{GV}$. By means of a statistical analysis of the spectra, two main structures were recognized:

- Both the $\mathrm{H}$ and He spectra gradually soften in the rigidity range $30-230 \mathrm{GV}$.

- The spectra exhibit a spectral hardening at $232_{-30}^{+35} \mathrm{GV}$ for $\mathrm{H}$ and $243_{-31}^{+27} \mathrm{GV}$ for $\mathrm{He}$.

The spectral changes could be a result of the diffusion of cosmic rays on a background plasma waves partially due to self-generation and partly due to externally generated turbulence from large spatial scales to smaller scales where cosmic rays can resonate. More details can be found in Ref [2].

Moreover, precision measurements of the electron component in the cosmic radiation provide important information about the origin and propagation of cosmic rays in the Galaxy. PAMELA published results regarding negatively charged electrons between 1 and 

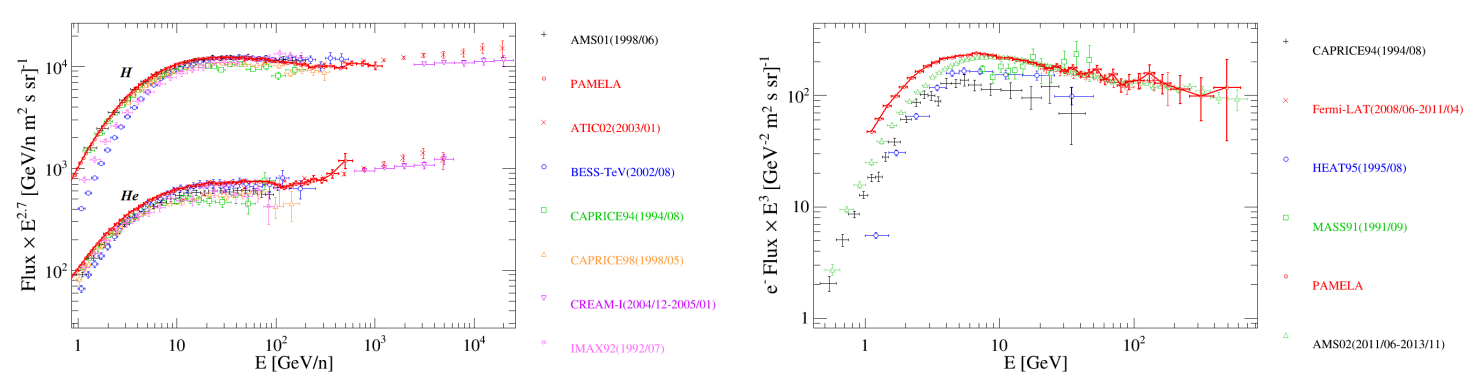

Figure 2: Proton and helium data (left) measured by PAMELA in the energy range from $1 \mathrm{GeV}$ to $\sim 1 \mathrm{TeV}$. Adapted from [1]. Electron data (right) up to $625 \mathrm{GeV}$. Adapted from [2].

$625 \mathrm{GeV}$; this is the first time that cosmic-ray $\mathrm{e}^{-}$have been identified above $50 \mathrm{GeV}$. Fig 2 (right) shows the electron flux up to $625 \mathrm{GeV}$. The data shows that the electron spectrum can be described with a single power-law energy dependence with spectral index -3.18 \pm 0.05 above the energy region influenced by the solar wind $(>30 \mathrm{GeV})$. No significant spectral features are observed and the data can be interpreted in terms of conventional diffusive propagation models. More details can be found in Ref [3]

\section{Boron and carbon nuclei}

PAMELA has measured the absolute fluxes of boron and carbon and the B/C ratio, which plays a central role in galactic propagation studies in order to derive the injection spectra at sources from measurements at Earth [4]. During the propagation in the interstellar medium (ISM) from the acceleration sites to Earth, galactic cosmic rays undergo various physical processes (there is still uncertainty in determining which are the most relevant physical processes) which shape the injection spectra and chemical compositions down to the measured ones at Earth. The spectra of particles which are produced exclusively by interactions of primary particles with ISM provide good constraints to propagation parameters. In particular, boron nuclei in cosmic rays are produced mainly by spallation of carbon with contributions from other elements. Thus, the boron-to-carbon ratio (B/C) has been extensively studied in order to tune propagation models. Spectra of boron and carbon are shown in Fig 3 (left), while the B/C ratio is shown in Fig 3 (right), in the kinetic energy range 0.44-129 GeV/n [4].

A good agreement with previous measurements can be observed, except at low energy where the effects of solar modulation are significant and must be carefully taken into account when comparing fluxes measured in different solar periods. The fitted spectral indexes above $20 \mathrm{GeV} / \mathrm{n}$ are $3.01 \pm 0.13$ for boron and $2.72 \pm 0.06$ for carbon. A very simple fit of PAMELA data based on the GALPROP transport code gives a slope of the diffusion coefficient $\circ 0.397 \pm 0.007$, thus not allowing to discriminate between Kolmogorov and a Kraichnan diffusion types. However, this has been obtained with a specific transport model, and may vary when using different models. 

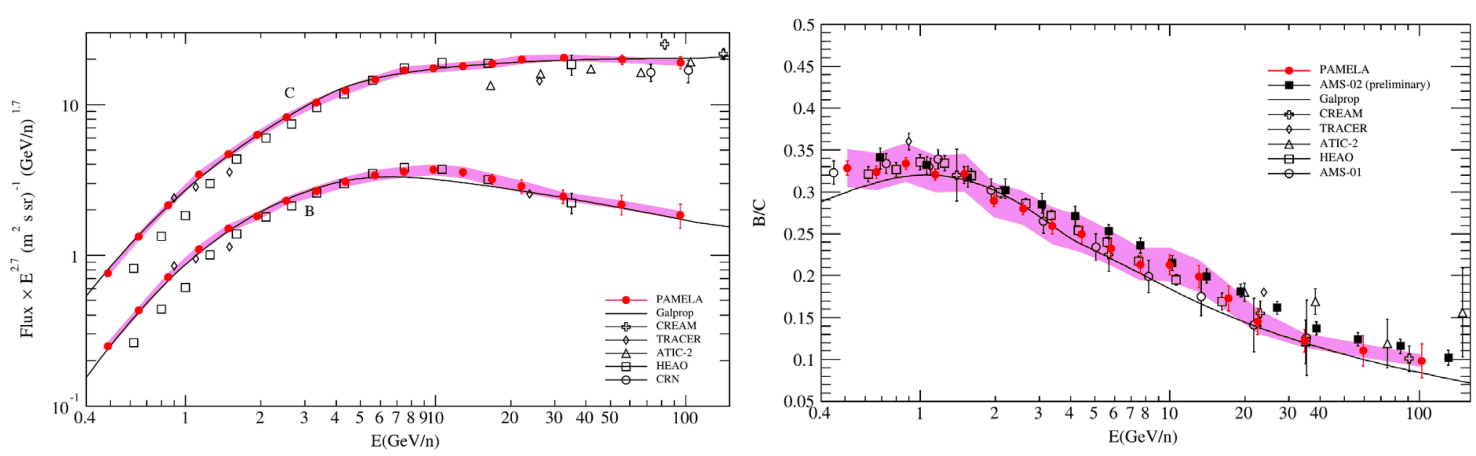

Figure 3: Boron and carbon fluxes multiplied by $E^{2.7}$ (left) and $B / C$ flux ratio (right) as measured by PAMELA. Adapted from [4].

\section{Lithium and beryllium nuclei}

$\mathrm{Li}, \mathrm{Be}$ (just like $\mathrm{B}$ ) in cosmic rays represent the lightest group of elements being of almost pure secondary origin. According to measurements, their relative abundance exceed that observed in normal matter by more than 4 order of magnitude and this is commonly interpreted as the effect of nuclear fragmentation of heavier nuclei during propagation from cosmic-ray sources to Earth. The constraint that the measurement of $\mathrm{Li}$ and $\mathrm{Be}$ abundances can provide to propagation models is still limited and as a matter of fact the available measurements of $\mathrm{Li}$ and Be species are scarce. The PAMELA identification capabilities will permit to measure Li and Be fluxes: these nuclei species are detected by the tracking system in a partially-saturated regime. However, both the tracking resolution and the charge separation based on multiple $\mathrm{dE} / \mathrm{dx}$ measurements by the tracker sensors are good enough to measure their absolute abundances up to high energy. In particular, for $\mathrm{Li}$ and Be identification the full redundant set of $\mathrm{dE} / \mathrm{dx}$ measurements provided by the PAMELA apparatus (TOF, tracking-system and calorimeter) can be used, with minor effects due to tracking-system detector saturation, to cleanly select these rare secondary elements against a large background of other particles. The average $\mathrm{dE} / \mathrm{dx}$ in the tracking system as a function of the inverse of the velocity $\beta$ measured by the TOF system is shown in Fig 4 before and after the saturated hits exclusion.

\section{Hydrogen, helium, lithium and beryllium isotopes}

Measurements of the isotopic composition of elements of the cosmic radiation provide significant constraints on cosmic ray source composition and cosmic ray transport and acceleration in the Galaxy. For example, the rare isotopes ${ }^{2} \mathrm{H}$ and ${ }^{3} \mathrm{He}$ in cosmic rays are generally believed to be of secondary origin, resulting mainly from the nuclear interactions of primary cosmic-ray protons and ${ }^{4} \mathrm{He}$ with the interstellar medium. Moreover, while the ${ }^{6} \mathrm{Li}$ isotope is a pure product of interactions of galactic cosmic rays with this interstellar medium, the ${ }^{7} \mathrm{Li}$ isotope has several sources. In addition to the production of ${ }^{7} \mathrm{Li}$ by spallation during the propagation of cosmic rays, the stellar production and primordial 

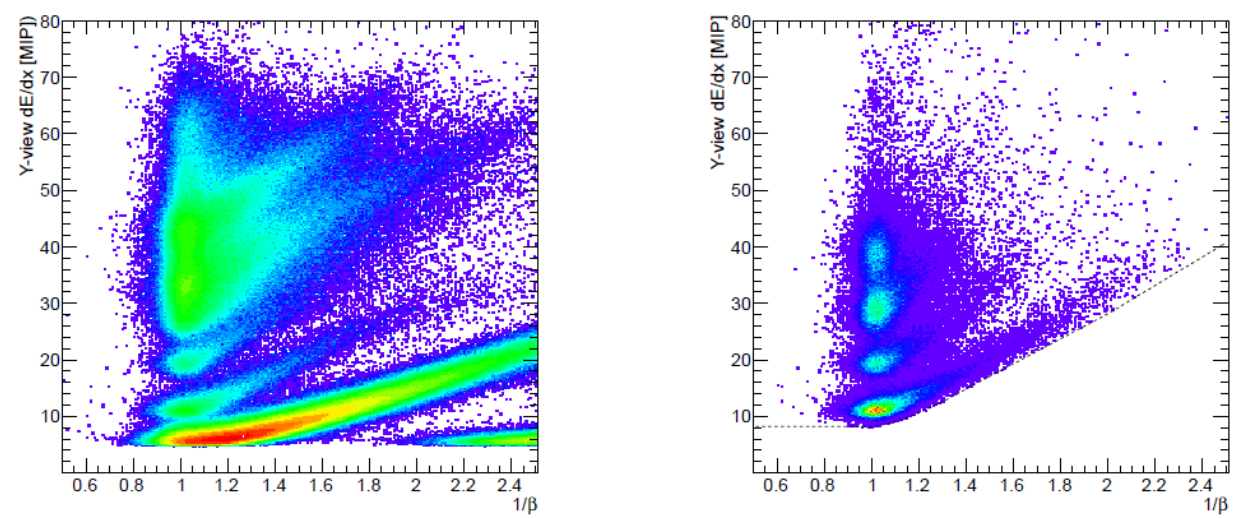

Figure 4: Average $d E / d x$ vs $1 / \beta$ in the tracking system evaluated by using hits from the ohmic side ( $\mathrm{Y}$ view) of the tracking sensors (left). On the right the same distribution is showed but after the saturated hits are excluded.

nucleosynthesis of ${ }^{7} \mathrm{Li}$ are possible sources. PAMELA measurements for ${ }^{2} \mathrm{H} /{ }^{1} \mathrm{H},{ }^{3} \mathrm{He} /{ }^{4} \mathrm{He}$, ${ }^{7} \mathrm{Li} /{ }^{6} \mathrm{Li}$ and ${ }^{7} \mathrm{Be} /\left({ }^{9} \mathrm{Be}+{ }^{10} \mathrm{Be}\right)$ are shown respectively in Fig 5 (left), Fig 5 (right), Fig 6 (left) and Fig 6 (right)
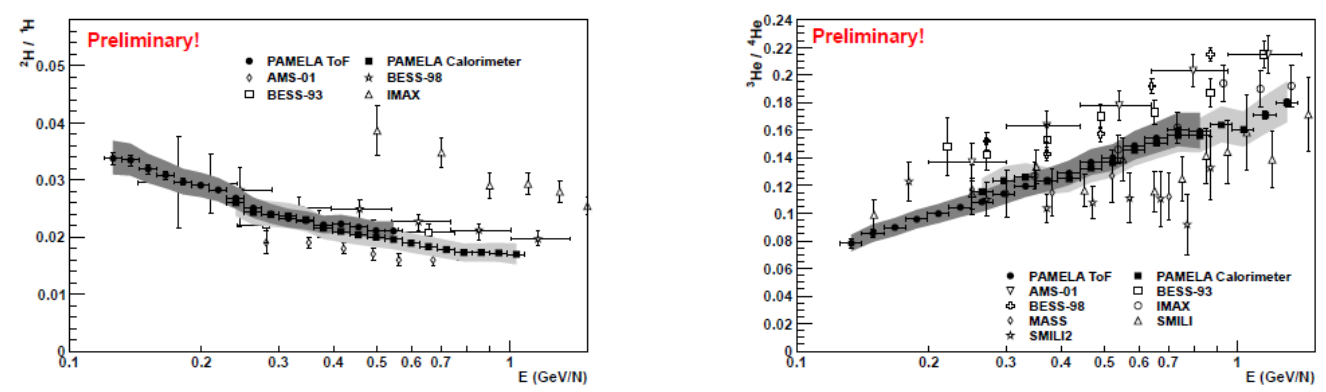

Figure 5: Results for the ratios ${ }^{2} \mathrm{H} /{ }^{1} \mathrm{H}$ (left) and ${ }^{3} \mathrm{He} /{ }^{4} \mathrm{He}$ (right) derived with the ToF or the calorimeter.

It is visible that the PAMELA results in Fig 5 are more precise in terms of statistics (this is due to the fact that other data, apart from the AMS-01 ones, are from balloon-borne experiments with limited data-taking and are effected by the non-negligible background of atmospheric secondary particle production). It is worth noticing that the PAMELA results obtained via the ToF analysis and via the multiple $\mathrm{dE} / \mathrm{dx}$ measurements with the calorimeter agree very well within their systematic errors. This gives confidence to the results. Also for what concerns results for the ratios ${ }^{7} \mathrm{Li} /{ }^{6} \mathrm{Li}$ and ${ }^{7} \mathrm{Be} /\left({ }^{9} \mathrm{Be}+{ }^{10} \mathrm{Be}\right)$, there is a good agreement between the ToF-derived measurements and the calorimeter-derived ones and with other experiments. 

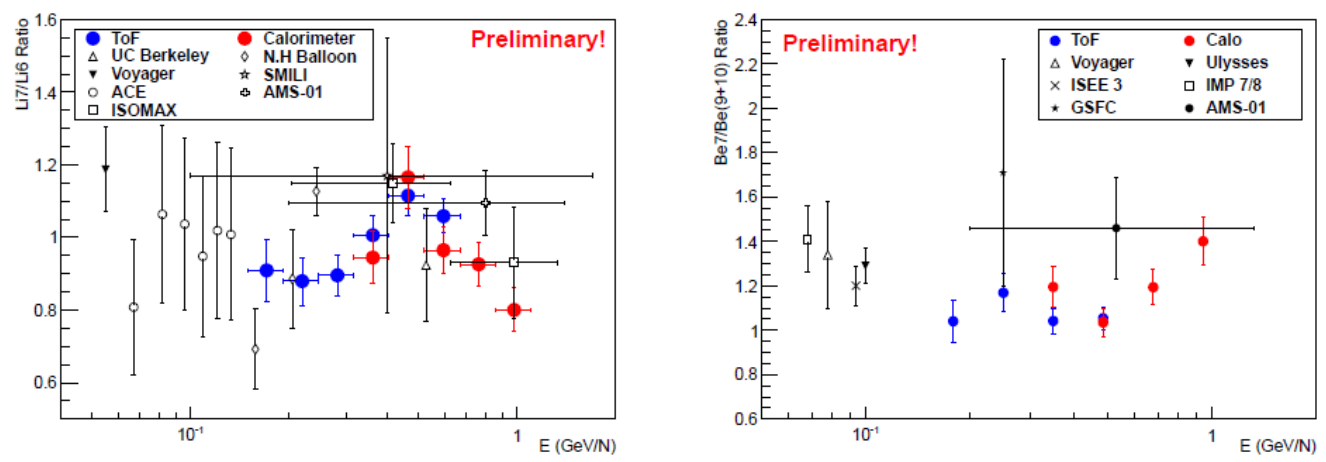

Figure 6: Results for the ratios ${ }^{7} \mathrm{Li} /{ }^{6} \mathrm{Li}$ (left) and ${ }^{7} \mathrm{Be} /\left({ }^{9} \mathrm{Be}+{ }^{10} \mathrm{Be}\right)$ (right) derived with the ToF or the calorimeter.

\section{7. $\overline{\mathrm{He}} / \mathrm{He}$ and search for SQM}

The explanation of the observed baryon asymmetry, i.e., the almost complete absence of antimatter in the visible part of the Universe, is one of the most important problems in cosmology. The real asymmetry value can be determined by direct measurements of the fluxes of antinuclei with charges $\mathrm{Z} \geq 2$ in primary cosmic rays near the Earth. The results of the search for antihelium using data from the PAMELA experiment (June 2006 - December 2009) showed no events with $Z=-2$ have been detected in the interval 0.6-600 GV. Thus, an upper limit on the $\overline{\mathrm{He}} / \mathrm{He}$ flux ratio has been set as a function of the energy and it is shown in Fig 7 (left). More details can be found in Ref [18]. PAMELA presented also some results for direct search of Strange Quark Matter in cosmic rays. If this state of matter exists it may be present in cosmic rays as particles, called strangelets, having an anomalously high mass-to-charge $(A / Z)$ ratio. A direct search in space is complementary in range and region to those coming from ground based spectrometers. In the energy range from 0.4 to $\sim 1200 \mathrm{GV}$, corresponding to a mass range from 10 to $\sim 105$ a.m.u., no such particles were found in the data and thus an upper limit as a function of baryon number has been set and it is shown in Fig 7 (right).

\section{The antiparticle puzzle}

Measurements of the presence of cosmic ray antiparticles (i.e. antiprotons and positrons), produced in the interaction between cosmic rays and the interstellar matter, address many questions in contemporary astrophysics, such as the nature and distribution of particle sources in our Galaxy, and the subsequent propagation of the particles through the whole Galaxy and the solar magnetosphere. Moreover, new sources of primary cosmic ray antiparticles of either astrophysical or exotic origin can be probed [1]. PAMELA measurements of antiproton-to-proton ratio, first published in Ref [5] are shown in Fig 8 (left), while data of the $\frac{e^{+}}{e^{+}+e^{-}}$(first published in Ref [6]) are shown in Fig 8 (right). 

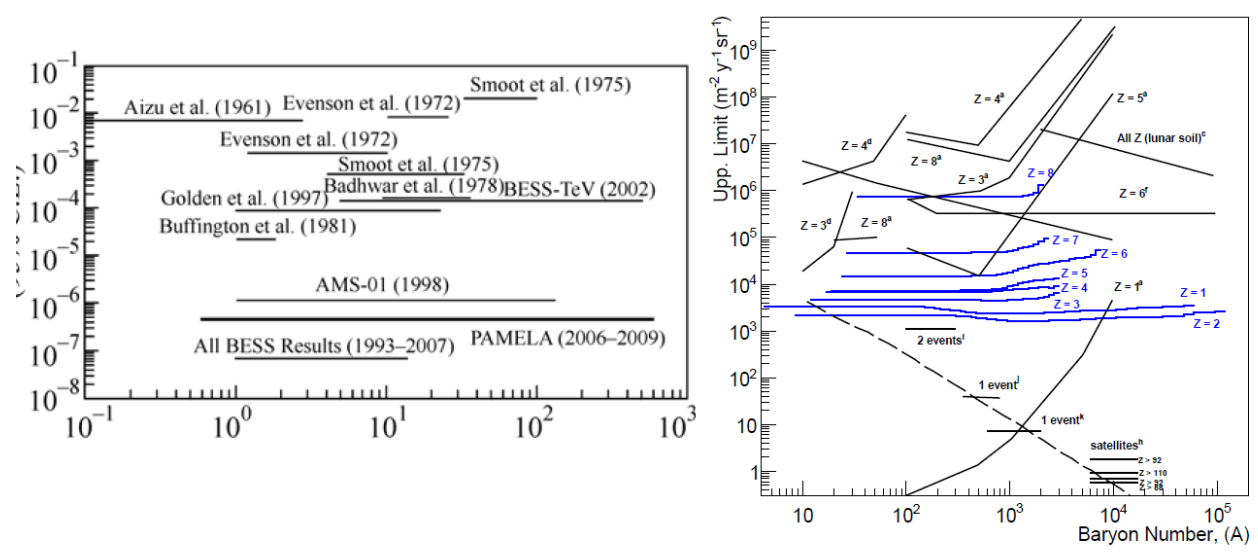

Figure 7: Upper limit on the $\overline{\mathrm{He}} / \mathrm{He}$ ratio (left), adapted from [18] and integral upper limits in terms of baryon number $A$ as measured by PAMELA for $1 \leq \mathrm{Z} \leq 8$ (right).
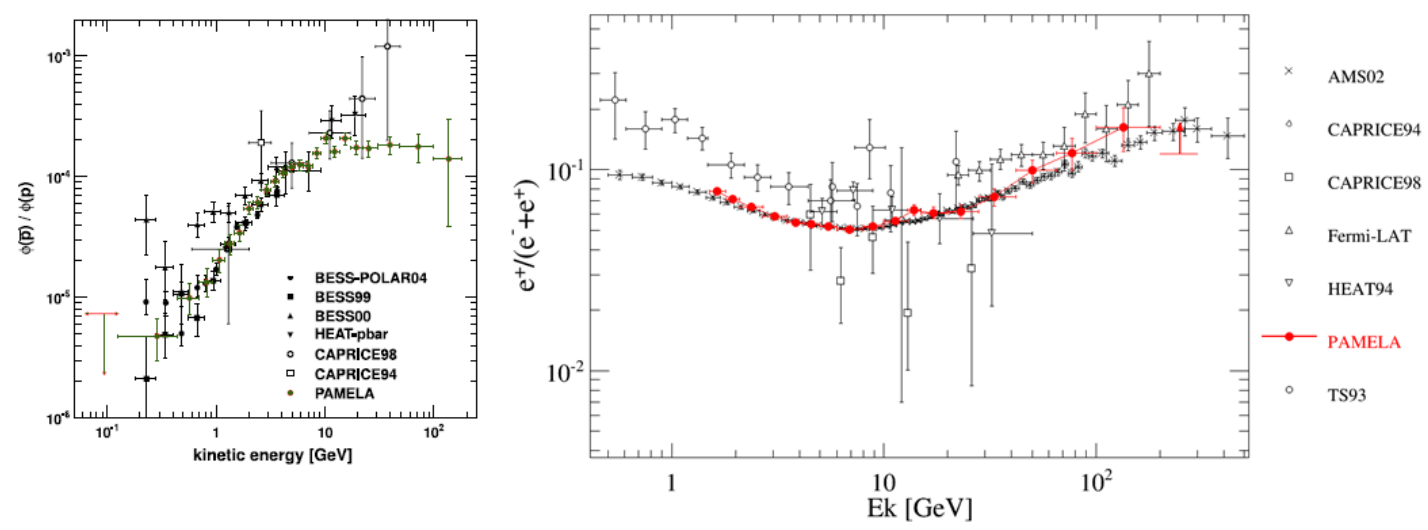

Figure 8: PAMELA results along with other recent measurements of the antiproton-to-proton flux ratio (left) and $\frac{e^{+}}{e^{+}+e^{-}}$(right). Adapted from [1].

PAMELA $\bar{p}$ data (up to January 2010) are good agreement, considering the uncertainties, with other data and they can be used to significantly constrain models of galactic Dark Matter such as those predicting heavy Weakly Interacting Massive Particle candidates. Also the low energy data are important because they can be used to constrain models with light $(\sim 10 \mathrm{GeV})$ Dark Matter candidates.

The resulting positron fraction measured by PAMELA between 1.5 and $300 \mathrm{GeV}$ along with results from other recent experimental cosmic-ray space and balloon-borne experiments is shown in Fig 8 (right). Two features are clearly visible in the data:

- Below $5 \mathrm{GeV}$ PAMELA results are systematically lower than other data.

- Above $10 \mathrm{GeV}$ they show that the positron fraction increases significantly with energy, opposite to the expectation for secondary production. 
The low energy discrepancy with data collected during the 1990s, i.e. from the previous solar cycle that favoured positively-charged particles, are interpreted as a consequence of solar modulation effects. At higher energies the agreement gives good confidence that the increase of the positron flux can be ascribed to a physical effect and not to systematic ones affecting the measurements. The high energy increase (that deviates from models has led to many speculations about a primary origin for the positrons. An excess in the positron population at high energy was postulated for the annihilation of Dark Matter particles in the galactic halo. While extremely intriguing, such an explanation is challenged by the asymmetry between the leptonic (positrons) and hadronic (antiprotons) PAMELA data. Such an asymmetry is difficult to explain in a framework where the neutralino is the dominant dark matter component. More detail can be found in Ref $[5,3,1]$.

\subsection{Anisotropies in $\mathrm{e}^{+}$and $\mathrm{e}^{-}$}

Cosmic rays have diffused inside the Galaxy for a very long time under the effect of magnetic fields and their arrival direction distribution is highly randomized. Since 80's has been clearly observed an energy-dependent anisotropy of galactic cosmic rays, both in the northern and in the southern hemisphere. Anisotropy studies can lead to a better understanding of cosmic ray propagation into the local interstellar medium. Furthermore, contrary to hadrons, cosmic ray electrons and positrons (CREs) lose rapidly their energy, due to synchrotron radiation emission and inverse Compton collisions with low-energy photons of the interstellar radiation field. These losses limit distances over which CREs can remain highly energetic. A way to distinguish between CRE origin (and also explain the excess of positrons above $10 \mathrm{GeV}$ ) is represented by the detection of an excess/deficit in their arrival direction. Astrophysical sources need to be relatively close at these energies, thus implying an anisotropy in the local CR positron flux. The detection techniques are described in Ref [7]. Fig 9 (left) shows the significance map obtained applying the technique described in Ref [8] on positron data for a $10^{\circ}$ integration radius. Fig 9 (right) shows the same thing but for electrons.
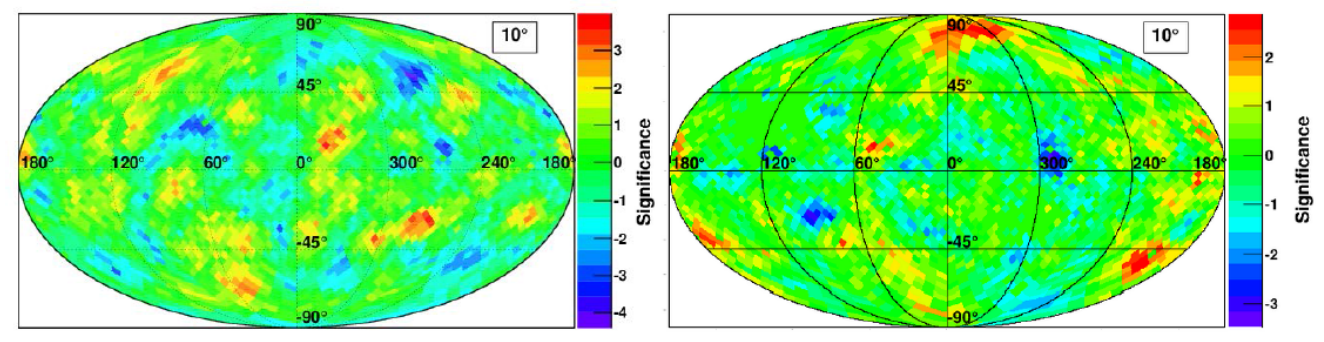

Figure 9: Significant maps for backtraced positrons (left) and electrons (right).

Results are consistent with isotropy at all angular scales considered. To take into account the effect of geomagnetic field particles have been backtraced up to 25 Earth's radii. The statistical analysis applied does not highlight significant deviation from the isotropy. 


\section{Time dependence of proton, positron and electrons fluxes}

The energy spectra of galactic cosmic rays, when measured near Earth, are significantly affected by the solar magnetic field. A comprehensive description of the cosmic radiation must therefore include the transport and modulation of cosmic rays inside the heliosphere. During the end of the last decade, the Sun underwent a peculiarly long quiet phase well suited to study modulation processes. PAMELA near-monthly (2006-2009) results on protons from $400 \mathrm{MV}$, compared with a state-of-the-art three-dimensional model of solar modulation, are presented in Ref [9] Other than protons, PAMELA observed the chargesign dependent solar modulation effects also on electrons [16] and positrons: the time variation of $\mathrm{e}^{-}, \mathrm{e}^{+}$, and $\mathrm{e}^{+} / \mathrm{e}^{-}$fraction is presented in Fig 10.
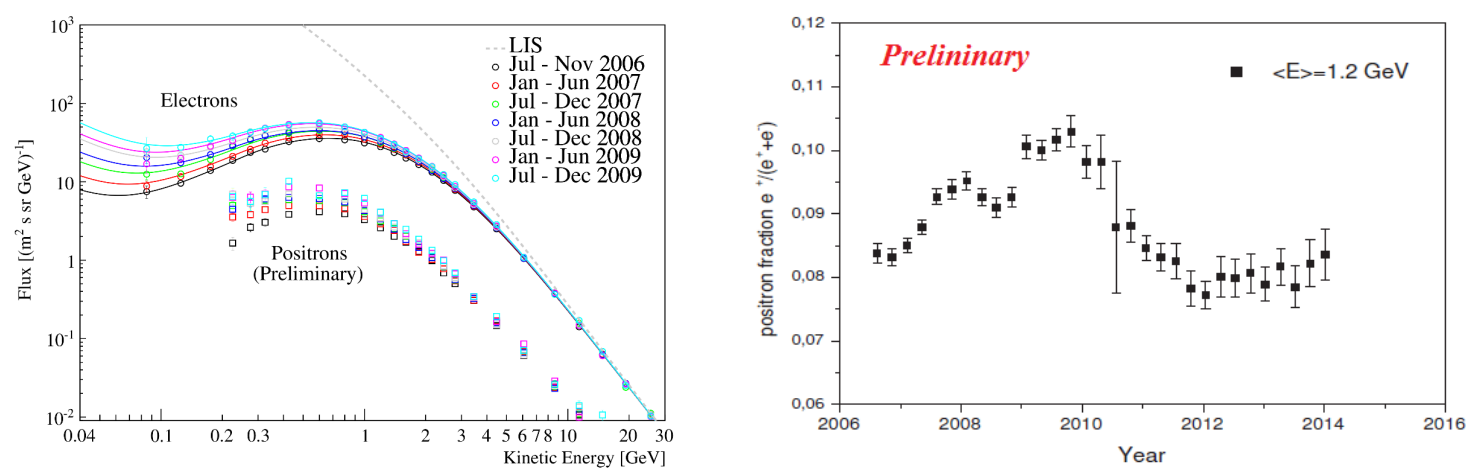

Figure 10: Time evolution of electron and positron fluxes (left) in the period 2006-2009 and time evolution of the $\mathrm{e}^{+} / \mathrm{e}^{-}$fraction as a function of time (right).

\section{Solar Particle Events}

The problem concerning the mechanism and site of Solar Energetic Particles (SEPs) acceleration remains an open question. SEPs may be produced after powerful explosive events on the Sun, accompanied by solar flares, coronal mass ejections, bursts of $X / \gamma$-rays and radio emission; it is clear that not a single mechanism is not involved in their formation. PAMELA started to observe SEPs fluxes during the 2006, December $13^{\text {th }} / 14^{\text {th }}$ events which also represent the first direct measurement of SEPs in space with a single instrument in the energy range from $\sim 80 \mathrm{MeVn}^{-1}$ to $\sim 3 \mathrm{GeVn}^{-1}$. Solar helium (up to $1 \mathrm{GeVn}^{-1}$ ) and protons (up to $\sim 2 \mathrm{GeVn}^{-1}$ ) were recorded. A study on the comparison between PAMELA data and Neutron Monitors, GOES and Ice Top was carried out, together with a deep study on the spectral shape fitting. More details can be found in Ref [10]. Since then PAMELA collected many data from other solar events, which are shown in Fig 11.

\section{Magnetospheric effects of high-energy SEPs}

Another great challenge in constraining scenarios for SEPs acceleration is due to the fact that the signatures of acceleration itself are heavily modified by transport within 


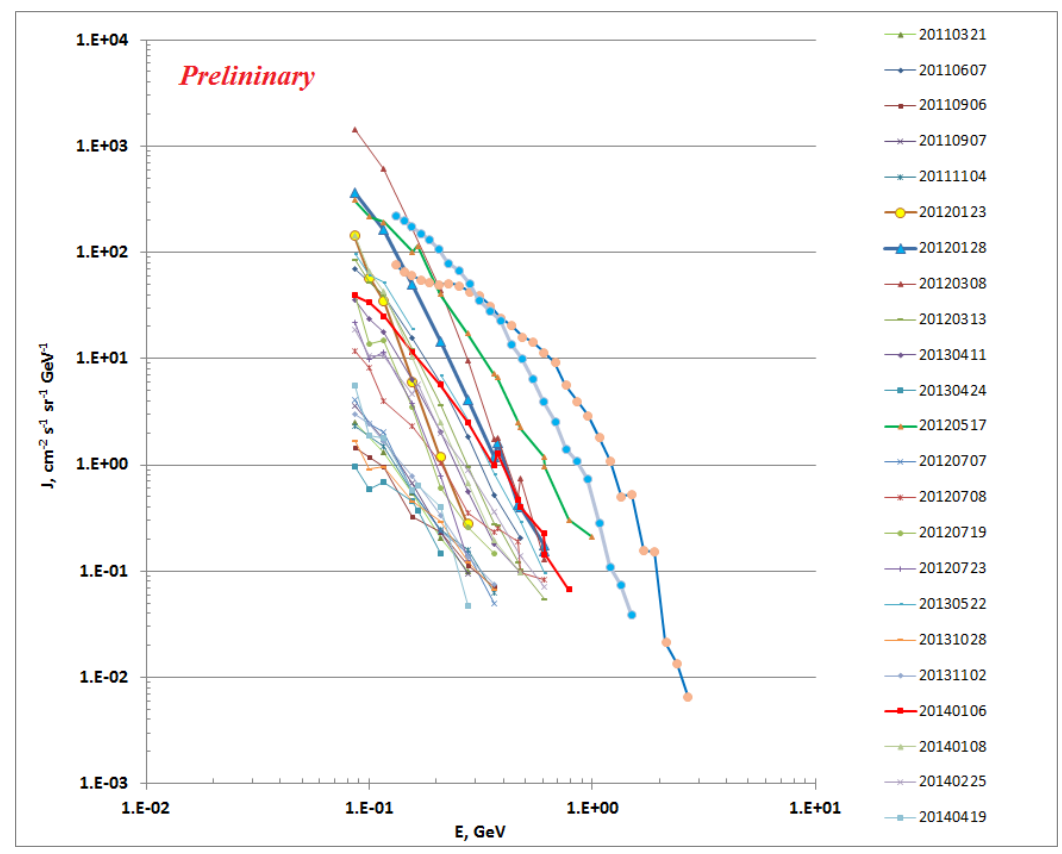

Figure 11: Pure solar proton spectra of all the events measured by PAMELA from 2006 up to September 2014.

interplanetary space. During transport, SEPs are subject to pitch angle scattering by the turbulent magnetic field, adiabatic focusing, or reflecting magnetic structures. Ground Level Enhancements (GLEs), such as the 2012 May $17^{\text {th }}$ one, provide an ideal way to study acceleration with minimal transport. PAMELA present in Ref [13] a unique high-energy SEP observation from the May event and interpret the observed pitch angle distributions as a result of local scattering ( $1 \mathrm{AU}$ ) by the Earth's magnetosheath. For these studies new analysis method were developed to estimate SEP energy spectra as a function of the particle asymptotic pitch angle. The crucial ingredient is provided by an accurate simulation of the asymptotic exposition of the PAMELA apparatus, based on a realistic reconstruction of particle trajectories in the Earth's magnetosphere. The results of this backtracing procedure are shown in Fig 12 (left), where the asymptotic cones of acceptance of the PAMELA apparatus for sample rigidities (evaluated in geographical coordinates) are depicted for the first PAMELA polar pass (0157-0220 UT) of May $17^{\text {th }}$. More detail on the procedure can be found in [12].

Fig 12 (right) shows PAMELA's pitch angle distribution (background subtracted) in 3 rigidity ranges. PAMELA observed two populations simultaneously with very different pitch angle distributions: a low-energy component (0.39-1.07 GV) confined to pitch angles $<90^{\circ}$ and a high-energy component (1.50-2.09 GV) that is beamed with pitch angles $<30^{\circ}$. Studies showed that this is the first observation of differential dispersion as particles transit the magnetosheath. More details can be found in [13]. 

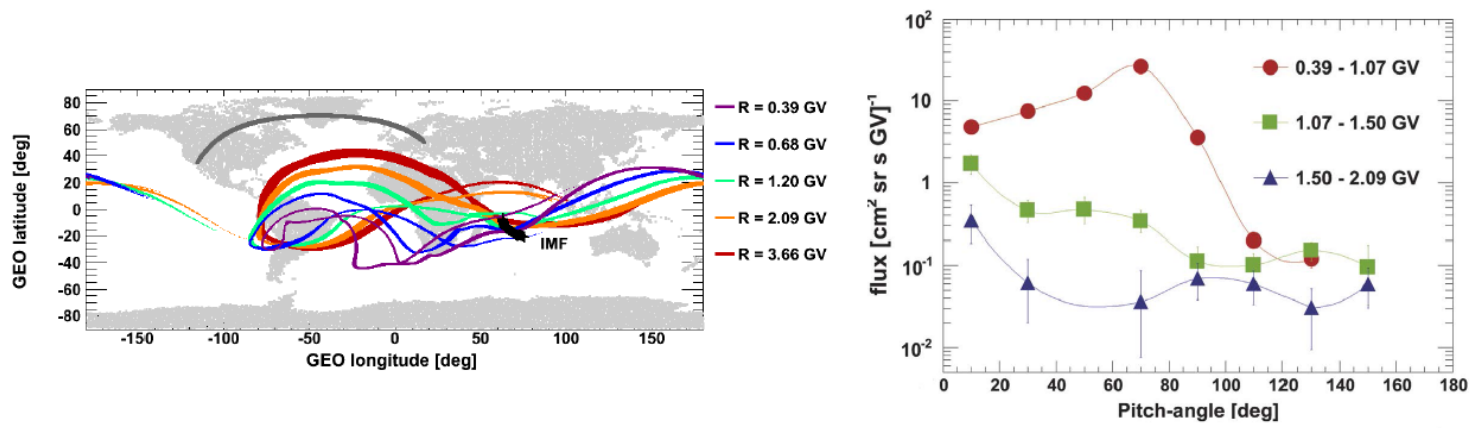

Figure 12: Asymptotic cones of acceptance of the PAMELA apparatus for sample rigidities for the first PAMELA polar pass (left). PAMELA's pitch angle distribution (background subtracted) in 3 rigidity ranges (right).

\section{Earth's magnetosphere: geomagnetically trapped and albedo particles}

Data from the PAMELA were also used to perform a detailed measurement of undercutoff protons at low Earth orbits. On the basis of a trajectory tracing approach using a realistic description of the magnetosphere, protons were classified into geomagnetically trapped and albedo. The former include stably-trapped protons in the South Atlantic Anomaly, which were analysed in the framework of the adiabatic theory, investigating energy spectra, spatial and angular distributions. The albedo protons were classified into quasi-trapped, concentrating in the magnetic equatorial region, and un-trapped, spreading over all latitudes and including both short-lived (precipitating) and long-lived (pseudotrapped) components. Precise measurements of electron and positron fluxes in energy range from $80 \mathrm{MeV}$ to several $\mathrm{GeV}$ below the geomagnetic cutoff rigidity and trapped/albedo cosmic ray deuteron fluxes were also carried out using the PAMELA magnetic spectrometer. The trapped proton results are depicted in Fig 13 (left), the $\mathrm{e}^{+} / \mathrm{e}^{-}$results in Fig 13 (center) and the trapped deuteron reconstructed trajectories in Fig 13 (right). More details can be found in Ref $[14,15,17]$

\section{References}

[1] O. Adriani et al., The PAMELA Mission: Heralding a new era in precision cosmic ray physics, Phys. Rep. 544 (2014) 323 [10.1016/j.physrep.2014.06.003].

[2] O. Adriani et al., PAMELA Measurements of Cosmic-Ray Proton and Helium Spectra, Science 332 (2011) 69 [10.1126/science.1199172].

[3] O. Adriani et al., Cosmic-ray electron flux measured by the PAMELA experiment between 1 and $625 \mathrm{GeV}$, Phys. Rev. Lett 106 (2011) 201101 [10.1103/PhysRevLett.106.201101].

[4] O. Adriani et al., Measurements of Boron and Carbon fluxes in cosmic rays with the PAMELA experiments, Astrophys. J. 791 (2014) 93 [10.1088/0004-637X/791/2/93]. 

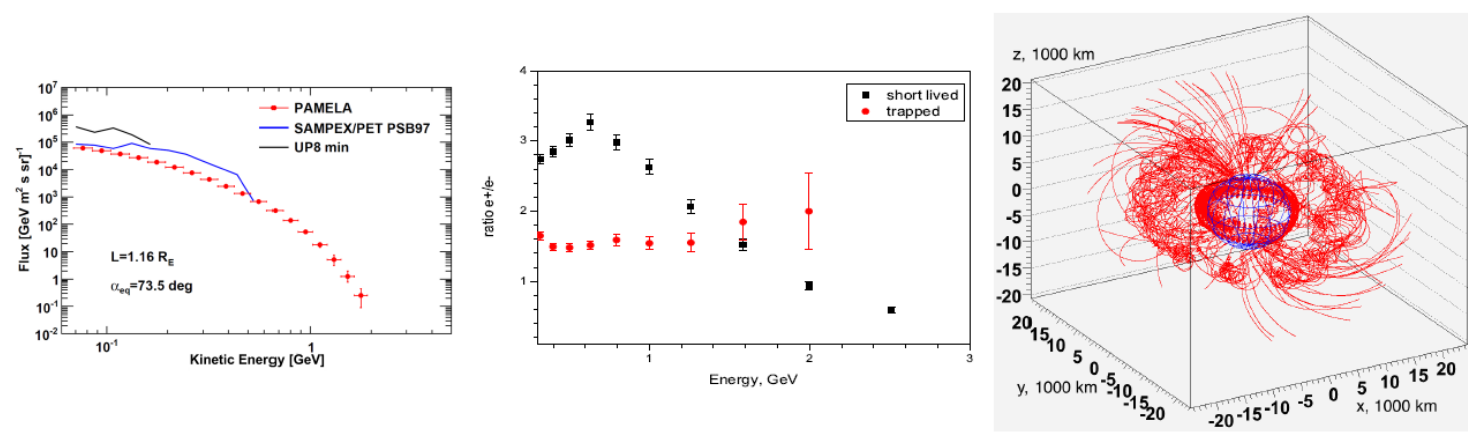

Figure 13: PAMELA trapped proton energy spectrum (left), $\mathrm{e}^{+} / \mathrm{e}^{-}$as a function of energy and reconstructed trajectories for deuterons of different populations.

[5] O. Adriani et al., PAMELA Results on the Cosmic-Ray Antiproton Flux from $60 \mathrm{MeV}$ to $180 \mathrm{GeV}$ in Kinetic Energy, Phys. Rev. Lett. 105 (2010) 121101 [10.1103/PhysRevLett.105.121101].

[6] O. Adriani et al., An anomalous positron abundance in cosmic rays with energies 1.5-100GeV, Nat. 458 (2009) 607 [10.1038/nature07942].

[7] B. Panico et al., A method to detect positron anisotropies with Pamela data, Nuc. Phys. B 256 (2014) 173 [10.1016/j.nuclphysbps.2014.10.020].

[8] O. Adriani et al., Search for Anisotropies in Cosmic-ray Positrons Detected By the PAMELA Experiment, ApJ 811 (2015) 21 [10.1088/1742-6596/409/1/012055].

[9] O. Adriani et al., Time Dependence of the Proton Flux Measured by PAMELA during the 2006 July-2009 December Solar Minimum, Astrophys. J. 765 (2013) 91 [10.1088/0004-637X/765/2/91].

[10] O. Adriani et al., Observations of the 2006 December 13 and 14 Solar Particle Events in the $80 \mathrm{MeVn}^{-1}-3 \mathrm{GeVn}^{-1}$ Range from Space with the PAMELA Detector, Astrophys. J. 742 (2011) 102 [10.1088/0004-637X/742/2/102].

[11] O. Adriani et al., Pamela's Measurements of Magnetospheric Effects On High Energy Solar Particles, Astrophys. J. Lett. 801 (2015) L3 [10.1088/2041-8205/801/1/L3].

[12] A. Bruno et al., Back-tracing and flux reconstruction for solar events with PAMELA, [arXiv:1412.1765].

[13] O. Adriani et al., Pamela's Measurements of Magnetospheric Effects On High Energy Solar Particles, Astrophys. J. Lett. 801 (2015) L3 [10.1088/2041-8205/801/1/L3].

[14] O. Adriani et al., Reentrant albedo proton fluxes measured by the PAMELA experiment, J. Geophphys. Res. 120 (2015) 3728 [10.1002/2015JA021019].

[15] O. Adriani et al., Trapped Proton Fluxes at Low Earth Orbits Measured by the PAMELA Experiment, Astrophys. J. Lett. 799 (2015) L4 [10.1088/2041-8205/799/1/L4].

[16] O. Adriani et al., Time Dependence of the $e^{-}$Flux Measured by PAMELA during the July 2006-December 2009 Solar Minimum, ApJ $\mathbf{8 1 0 ~ ( 2 0 1 5 ) ~} 142$ [10.1088/1742-6596/632/1/012073]. 
[17] S.A. Koldobskiy et al., Study of deuteron spectra under radiation belt with PAMELA instrument, JPCS 632 (2015) 012060 [10.1088/1742-6596/632/1/012060].

[18] A.G. Mayorov et al., Upper limit on the antihelium flux in primary cosmic rays, JETP Lett. 93 (2011) 628 [10.1134/S0021364011110087]. 